\title{
Whole Transcriptomic Analysis of Apigenin on TNFa Immuno-activated MDA-MB-231 Breast Cancer Cells
}

\author{
DAVID BAUER, ELIZABETH MAZZIO and KARAM F.A. SOLIMAN \\ College of Pharmacy and Pharmaceutical Sciences, Florida A\&M University, Tallahassee, FL, U.S.A.
}

\begin{abstract}
Background: Triple-negative breast cancer is categorized by a lack of hormone receptors, inefficacy of antiestrogen or aromatase inhibitor chemotherapies and greater mortality rates in African American populations. Advancedstage breast tumors have a high concentration of tumor necrosis factor- $\alpha(T N F \alpha)$ throughout the tumor/stroma milieu, prompting sustained release of diverse chemokines (i.e. C-C motif chemokine ligand 2 (CCL2)/CCL5). These potent chemokines can subsequently direct mass infiltration of leukocyte sub-populations to lodge within the tumor, triggering a loss of tumor immune surveillance and subsequent rapid tumor growth. Previously, we demonstrated that in the MDA$M B-231$ TNBC cell line, TNF $\alpha$ evoked a rise in immune signaling proteins: CCL2, granulocyte macrophage colonystimulating factor, interleukin (IL)1 $\alpha$, IL6 and inhibitor of nuclear factor kappa-B kinase subunit epsilon (IKBKE) all of which were attenuated by apigenin, a dietary flavonoid found in chamomile and parsley. Materials and Methods: The present work elucidates changes evoked by TNF $\alpha$ in the presence or absence of apigenin by examining the entire transcriptome for $m R N A$ and long intergenic non-coding RNA with Affymetrix Hugene-2.1_ST human microarrays. Differential geneexpression analysis was conducted on 48,226 genes. Results: $T N F \alpha$ caused up-regulation of 75 genes and down-regulation of 10. Of these, apigenin effectively down-regulated 35 of the 75 genes which were up-regulated by TNF $\alpha$. These findings confirm our previous work, specifically for the TNF $\alpha$-evoked spike in ILIA vs. untreated controls [+21-fold change (FC), $p<0.0001]$ being attenuated by apigenin in the presence of
\end{abstract}

This article is freely accessible online.

Correspondence to: Karam F.A. Soliman, Ph.D., College of Pharmacy and Pharmaceutical Sciences, Florida A\&M University, Dyson Pharmacy Building, Room 104, 1520 Martin Luther King Jr., Blvd, Tallahassee, FL 32307, U.S.A. Tel: +1 8505993306, Fax: +18505993667, e-mail: karam.soliman@famu.edu

Key Words: Triple-negative breast cancer, apigenin, $\mathrm{TNF} \alpha$, differential gene expression analysis, immune signaling.
TNFa (-15 FC vs. TNF $\alpha, p<0.0001)$. Similar trends were seen for apigenin-mediated down-regulation of TNF $\alpha$-up-regulated transcripts: IKBKE (TNF $\alpha$ : 4.55 FC vs. control, $p<0.001$; and TNF $\alpha$ plus apigenin: $-4.92 \mathrm{FC}, p<0.001)$, CCL2 (2.19 FC, $p<0.002$; and -2.12 FC, $p<0.003)$, IL6 (3.25 FC, $p<0.020$; and $-2.85 F C, p<0.043)$ and CSF2 (TNF $\alpha+6.04 F C$, $p<0.001$; and $-2.36 F C, p<0.007)$. In addition, these data further establish more than a $65 \%$ reduction by apigenin for the following transcripts which were also up-regulated by TNFa: cathepsin S (CTSS), complement C3 (C3), laminin subunit gamma 2 (LAMC2), (TLR2), toll-like receptor $2 G$ protein-coupled receptor class $C$ group 5 member $B$ (GPRC5B), contactin-associated protein 1 (CNTNAP1), claudin 1 (CLDN1), nuclear factor of activated T-cells 2 (NFATC2), C-X-C motif chemokine ligand 10 (CXCL10), CXCL11, interleukin 1 receptor-associated kinase 3 (IRAK3), nuclear receptor subfamily 3 group $C$ member 2 (NR3C2), interleukin 32 (IL32), IL24, slit guidance ligand 2 (SLIT2), transmembrane protein 132A (TMEM132A), TMEM171, signal transducing adaptor family member 2 (STAP2), mixed lineage kinase domain-like pseudokinase (MLKL), kinase insert domain receptor (KDR), BMP-binding endothelial regulator (BMPER), and kelch-like family member 36 (KLHL36). Conclusion: There is a possible therapeutic role for apigenin in down-regulating diverse genes associated with tumorigenic leukocyte sub-population infiltration by triple-negative breast cancer. The data have been deposited into the Gene Expression Omnibus for public analysis at https://www.ncbi. nlm.nih.gov/geo/query/acc.cgi? acc $=$ GSE120550.

Breast cancer is often sub-categorized as being either hormone receptor-positive or -negative, to which the latter group if devoid of estrogen and progesterone receptors and human epidermal growth factor receptor 2 (HER2) is further classified as triple-negative breast cancer (TNBC). TNBC is non-responsive to estrogen receptor modulators (e.g. raloxifene, tamoxifen) and aromatase inhibitors, can be quite aggressive and is often associated with lower overall survival odds, particularly in African Americans (1). While the standard treatment for TNBC includes surgery, radiation and various types of chemotherapies (e.g. taxanes, doxorubicin, 
or docetaxel/cyclophosphamide), new drug approaches or use of effective complementary and alternative medicines are needed. Possible drug candidates to treat aggressive TNBC are those that target phosphoinositide 3-kinase (PI3K), poly (ADP-ribose) polymerase (PARP), luminal androgen receptor (LAR) (2, 3), cytokine modulators, vaccines (4) and immune-therapies such as checkpoint antagonistic antibodies against programmed cell death-1 receptor/programmed death-ligand $1(5,6)$.

The immune system plays a critical role in both risk and mortality outcome associated with diverse human cancer types including TNBC. Initially, immune deficiency in immunocompromised individuals can play an essential role in tumor development. While a healthy immune system can target and destroy cancer cells through MHC class I chain-related molecules (MICs), cluster of differentiation (CD) $4^{+} / \mathrm{CD}^{+} \mathrm{T}$ - and natural killer cell activities, immunosuppression can hamper these biological responses and amplify the risk of developing spontaneous or carcinogen-induced tumors (7-10). Once a tumor is established, continual evasion of the immune system is part of malignant disease, carried out by the actions of chemotactic tumor-promoting proteins [e.g. tumor necrosis factor- $\alpha(\mathrm{TNF} \alpha)$, and $\mathrm{C}-\mathrm{C}$ motif chemokine ligand 2 (CCL2)] that direct inward migration of leukocyte subpopulations (LSPs) bearing CCR2/CCR5 receptors to the tumor microenvironment. Types of LSPs can include tumorassociated macrophages, tumor-associated neutrophils, T-regulatory cells, myeloid-derived suppressor cells, metastasis-associated macrophages and cancer-associated fibroblasts, all of which collectively foster tumor growth, stem cell survival, angiogenesis, metastasis (11) and overexpression of programmed cell death 1 and programmed death ligand 1 (12), all of which inactivate the normal tumor immune response (13-17).

Apigenin is a natural compound which has the capacity to antagonize several mitigating events in human tumor development and tumor immune evasion. These include the capability to reduce inflammation, inhibit casein kinase 2 (CK2), matrix metalloproteinases 9 and 1 , protein kinase $\mathrm{C}$ (18), cytochrome P450 IAI (19) topoisomerase I (20) tumor growth factors (21), CCL2-induced release by $\mathrm{TNF} \alpha-$ activated breast cancer (22) and blockade of drug resistanceassociated extrusion pumps $(23,24)$. In the current study, we expand on our previous work by evaluating the effects of apigenin on TNF $\alpha$-treated MDA-MB-231 cells (22) by analyzing the whole transcriptome.

\section{Materials and Methods}

Cell line, chemicals, and reagents. TNBC MDA-MB-231 cells were obtained from the American Type Culture Collection (Rockville, MD, USA). Dulbecco's modified Eagle's medium (DMEM), fetal

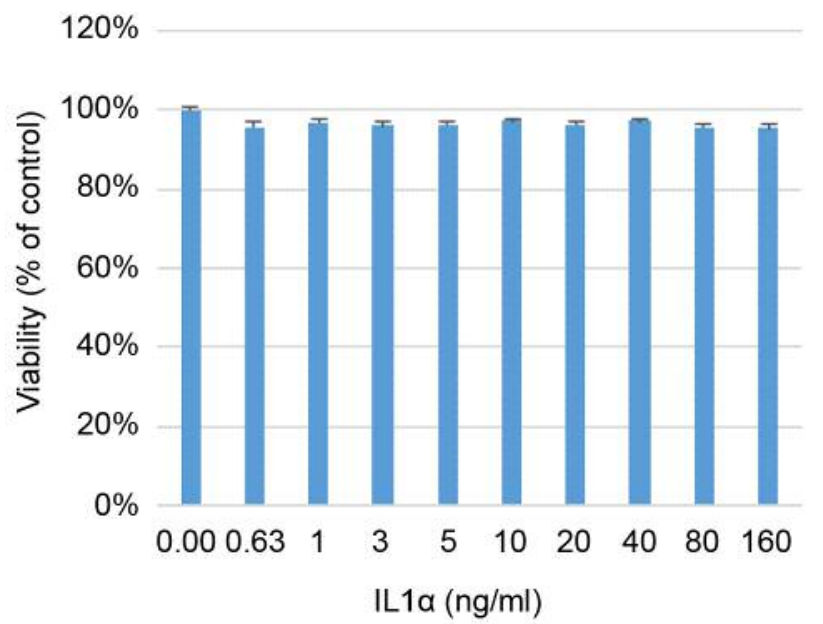

Figure 1. The effect of interleukin $1 \alpha(L 1 \alpha)$ on viability of MDA-MB231 cells at $5 \% \mathrm{CO}_{2} / \mathrm{Atm}$ for $24 \mathrm{~h}$. The data are the mean \pm S.E.M. $(n=4)$ and presented as viability relative to that of the control. No significant differences from the control were found by one-way ANOVA.

bovine serum (FBS), and penicillin/streptomycin were obtained from Invitrogen (Carlsbad, CA, USA). Recombinant human TNF $\alpha$ was purchased from RayBiotech (RayBiotech Inc., Norcross, GA, USA). Apigenin, CCL2 enzyme-linked immunosorbent assay (ELISA) kits, and recombinant IL1 $\alpha$ were purchased from Abcam (Cambridge, MA, USA).

Cell culture. MDA-MB-231 cells were grown in high-glucose DMEM (with phenol red and glutamine) then supplemented with 10\% FBS and $1 \%(10,000 \mathrm{U} / \mathrm{ml})$ penicillin $\mathrm{G}$ sodium and $(10,000 \mu \mathrm{g} / \mathrm{ml})$ streptomycin sulfate. Cells were grown at $37^{\circ} \mathrm{C}$ with humidified $95 \%$ air $/ 5 \% \mathrm{CO}_{2}$ and sub-cultured every 3-5 days.

Cell viability assay. Cell viability was determined using the Alamar Blue assay. Briefly, 96-well plates were seeded with MDA-MB-231 cells at a density of $5 \times 10^{4}$ cells/ $100 \mu \mathrm{l} /$ well. After $24 \mathrm{~h}$, Alamar blue $(0.1 \mathrm{mg} / \mathrm{ml}$ in Hank's balanced salt solution (HBSS) ) was added at $15 \% \mathrm{v} / \mathrm{v}$ to each well and plates were incubated for a further 6-8 $\mathrm{h}$. Quantitative analysis of dye conversion was measured using a Biotek Synergy multi-mode detection reader equipped with Gen5 software at 550/580 nm (excitation/emission) (BioTek Instruments, Inc., Winooski, VT, USA). All data are expressed as a percentage of live untreated controls.

CCL2 detection by ELISA. Supernatants from controls and TNF $\alpha-$ stimulated $(24 \mathrm{~h})$ MDA-MB-231 cells were collected and centrifuged at $100 \times \mathrm{g}$ for $5 \mathrm{~min}$ at $4^{\circ} \mathrm{C}$. ELISA was performed using MCP-1/CCL2 Human MCP1 ELISA Kit from Abcam (ab179886) (Cambridge, MA, USA) following the manufacturer's instructions. Briefly, $100 \mu \mathrm{l}$ of supernatant from samples were added to 96-well plates pre-coated with the capture antibody, followed by a wash, second antibody, detection antibody and final reading at 450 $\mathrm{nm}$ using a Biotek Synergy multi-mode detection reader equipped with Gen5 software (BioTek Instruments, Inc.). 


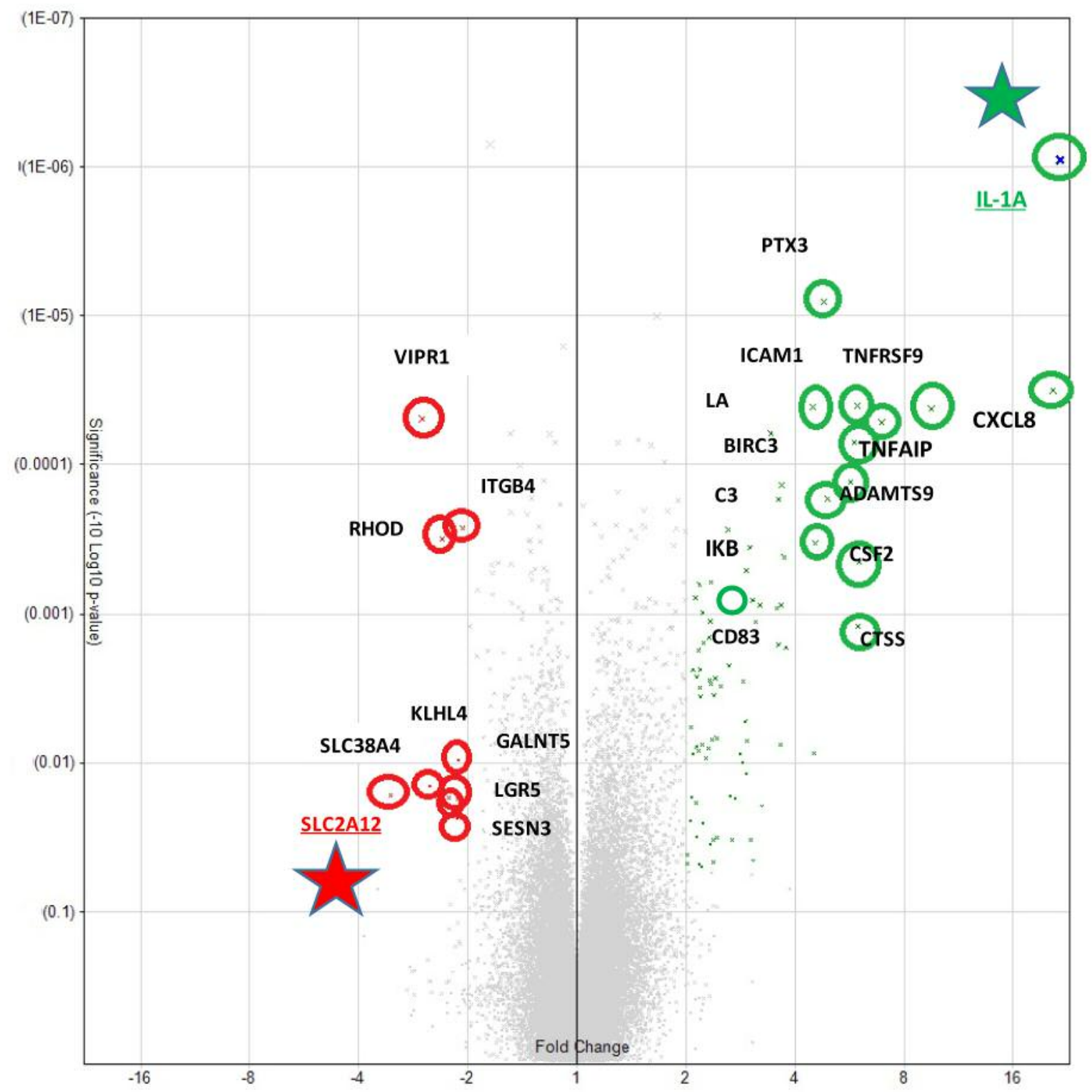

Figure 2. Whole transcriptomic differential changes between MDA-MB-231 cells after 24 h of treatment with tumor necrosis factor- $\alpha$ (TNF $\alpha)$ (40 $n g / m l)$. The data are presented as fold change $(F C)$ and ANOVA p-value. Up-regulated transcripts are shown in the right-hand panel, and downregulated transcripts are shown in the left-hand panel. Top differentially expressed genes are denoted by official gene symbol, with all data presented for differential changes in Table I.

Reverse transcription polymerase chain reaction (RT-PCR). MDA-MB231 cells were lysed with $1 \mathrm{ml}$ Trizol reagent. Chloroform $(0.2 \mathrm{ml})$ was added to the samples, which were then vortexed, incubated at $15-30^{\circ} \mathrm{C}$ for $2-3 \mathrm{~min}$ and centrifuged at $10,000 \times \mathrm{g}$ for $15 \mathrm{~min}$ at $2-8^{\circ} \mathrm{C}$. The aqueous phase was then transferred to a fresh tube, and the RNA precipitated by mixing $0.5 \mathrm{ml}$ isopropyl alcohol. RNA was extracted and subject to iScript advanced RT to the reaction. The reverse transcription was performed for $30 \mathrm{~min}$ at $42^{\circ} \mathrm{C}$ and $\mathrm{RT}$ inactivation for $5 \mathrm{~min}$. at $85^{\circ} \mathrm{C}$. For PCR reaction, the following components were mixed in a $0.5 \mathrm{ml}$ PCR tube: $5.0 \mu \mathrm{l}$ cDNA product, $10 \mu \mathrm{l}$ SsoAdvanced Universal SYBR Green Supermix (Bio-Rad Laboratories, Inc., Hercules, CA, USA), $1.0 \mu \mathrm{l}$ inhibitor of nuclear factor kappa-B kinase subunit epsilon $(K B K E)$ primer and $4 \mu \mathrm{l}$ water. PCR was performed with 39 cycles of denaturation: $15 \mathrm{~s}$ at $95^{\circ} \mathrm{C}$; annealing: $30 \mathrm{~s}$ at $60^{\circ} \mathrm{C}$; and extension $60 \mathrm{~s}$ at $72^{\circ} \mathrm{C}$ using a Bio-Rad CFX96 Real-Time System. cDNA synthesis and real-time PCR was performed using iScript ${ }^{\mathrm{TM}}$ advanced cDNA synthesis kit/advanced Universal SYBR ${ }^{\circledR}$ RT-PCR (Bio-Rad Laboratories, Inc.) was run and normalized to expression of glyceraldehyde-3-phosphate dehydrogenase (GAPDH) mRNA for each sample.

Microarray WT 2.1 human datasets. After treatment, cells were washed three times in ice-cold HBSS, rapidly frozen and stored at $-80^{\circ} \mathrm{C}$. Total RNA was isolated and purified using the TRIzol/ 
Table I. Differential whole transcriptome pattern induced in MDA-MB-231 cells by tumor necrosis factor- $\alpha$ (TNF $\alpha)(40 \mathrm{ng} / \mathrm{ml}) \mathrm{relative}$ to that of untreated controls. The data are presented as average $(n=3)$ bi-weight $\times$ signal $(\log 2)$.

\begin{tabular}{|c|c|c|c|c|}
\hline \multirow[b]{2}{*}{ Gene symbol } & \multirow[b]{2}{*}{ Description } & \multicolumn{3}{|c|}{ Expression } \\
\hline & & $\mathrm{TNF} \alpha$ & Control & FC \\
\hline$I L 1 A^{*}$ & Interleukin 1 alpha & 6.26 & 1.83 & 21.57 \\
\hline$C X C L 8^{*}$ & Chemokine (C-X-C motif) ligand 8 & 7.57 & 3.2 & 20.61 \\
\hline TNFAIP3 & Tumor necrosis factor, alpha-induced protein 3 & 6.76 & 3.51 & 9.51 \\
\hline TNFRSF9 & Tumor necrosis factor receptor superfamily, member 9 & 5.89 & 3.1 & 6.94 \\
\hline$C S F 2 *$ & Colony stimulating factor 2 (granulocyte-macrophage) & 5.07 & 2.47 & 6.04 \\
\hline CTSS* $^{*}$ & Cathepsin S & 8.38 & 5.8 & 5.97 \\
\hline ICAMI & Intercellular adhesion molecule 1 & 9.33 & 6.76 & 5.95 \\
\hline BIRC3 & Baculoviral iap repeat containing 3 & 8.84 & 6.3 & 5.84 \\
\hline ADAMTS9* & Adam metallopeptidase with thrombospondin type 1 motif 9 & 7.05 & 4.54 & 5.69 \\
\hline$C 3^{*}$ & Complement component 3 & 4.67 & 2.37 & 4.92 \\
\hline PTX3 & Pentraxin 3 , long & 9.03 & 6.76 & 4.81 \\
\hline$I K B K E^{*}$ & Inhibitor of kappa light polypeptide gene enhancer in B-cells, kinase epsilon & 6.58 & 4.39 & 4.55 \\
\hline$L A M C 2 *$ & Laminin, gamma 2 & 8.6 & 6.43 & 4.48 \\
\hline CD83 & CD83 molecule & 5.95 & 4.03 & 3.78 \\
\hline$T L R 2 *$ & Toll-like receptor 2 & 5.76 & 3.86 & 3.73 \\
\hline TRAF1 & Tnf receptor-associated factor 1 & 4.98 & 3.1 & 3.68 \\
\hline$G P R C 5 B^{*}$ & $\mathrm{G}$ protein-coupled receptor, class $\mathrm{c}$, group 5 , member b & 5.72 & 3.84 & 3.66 \\
\hline$N F K B 2$ & Nuclear factor of kappa light polypeptide gene enhancer in b-cells 2 (p49/p100) & 6.06 & 4.19 & 3.65 \\
\hline CNTNAP1* & Contactin associated protein 1 & 5.01 & 3.17 & 3.6 \\
\hline$C L D N 1^{*}$ & Claudin 1 & 5.03 & 3.18 & 3.6 \\
\hline IRAK2 & Interleukin 1 receptor-associated kinase 2 & 5.85 & 4.02 & 3.56 \\
\hline TNIP1 & Tumor necrosis factor, alpha-induced protein 1 & 7.59 & 5.81 & 3.43 \\
\hline IL6* & Interleukin 6 & 4.7 & 3 & 3.25 \\
\hline NUAK2 & NUAK family kinase 2 & 5.06 & 3.37 & 3.21 \\
\hline NFKBIE & Nuclear factor of kappa light polypeptide gene enhancer in B-cells inhibitor, epsilon & 6.6 & 4.96 & 3.12 \\
\hline SNORD18C; RPL4 & Small nucleolar RNA, C/D box 18c; ribosomal protein 14 & 5.24 & 3.62 & 3.07 \\
\hline NFKBIA & Nuclear factor of kappa light polypeptide gene enhancer in b-cells inhibitor, alpha & 8.06 & 6.45 & 3.06 \\
\hline NFATC $2 *$ & Nuclear factor of activated T-cells, cytoplasmic, calcineurin-dependent 2 & 5.46 & 3.87 & 3.01 \\
\hline SERPINB2; SERPINB10* & Serpin peptidase inhibitor, clade B (ovalbumin), member 2, and member 10 & 5.35 & 3.76 & 3.01 \\
\hline LOC100287834 & Uncharacterized LOC100287834 & 10.9 & 9.34 & 2.94 \\
\hline CXCL10* & Chemokine (C-X-C motif) ligand 10 & 3.21 & 1.66 & 2.94 \\
\hline CXCL11* & Chemokine (C-X-C motif) ligand 11 & 4.36 & 2.81 & 2.93 \\
\hline ERN1 & Endoplasmic reticulum to nucleus signaling 1 & 4.81 & 3.26 & 2.92 \\
\hline C15orf48; MIR147B & Chromosome 15 open reading frame 48 ; microRNA $147 \mathrm{~b}$ & 5.63 & 4.1 & 2.88 \\
\hline KRTAP2-3 & Keratin-associated protein $2-3$ & 4.86 & 3.34 & 2.87 \\
\hline LOC541472 & Uncharacterized LOC541472 & 2.98 & 1.48 & 2.83 \\
\hline $\operatorname{IRAK} 3^{*}$ & Interleukin 1 receptor-associated kinase 3 & 4.3 & 2.85 & 2.73 \\
\hline$N R 3 C 2 *$ & Nuclear receptor subfamily 3 , group $\mathrm{C}$, member 2 & 4.57 & 3.15 & 2.68 \\
\hline$I L 32^{*}$ & Interleukin 32 & 4.47 & 3.07 & 2.63 \\
\hline SERPINE1 & Serpin peptidase inhibitor, clade E (nexin, plasminogen activator inhibitor type 1), member 1 & 10.11 & 8.73 & 2.61 \\
\hline IL24* & Interleukin 24 & 5.14 & 3.83 & 2.49 \\
\hline$D R A M 1^{*}$ & DNA-damage regulated autophagy modulator 1 & 4.44 & 3.15 & 2.44 \\
\hline TNFAIP2 & Tumor necrosis factor, alpha-induced protein 2 & 5.43 & 4.14 & 2.44 \\
\hline TNFSF $15^{*}$ & Tumor necrosis factor (ligand) superfamily, member 15 & 4.02 & 2.75 & 2.41 \\
\hline SLIT2* & Slit guidance ligand 2 & 6.22 & 4.97 & 2.39 \\
\hline$C D 44$ & CD44 molecule (Indian blood group) & 4.53 & 3.28 & 2.38 \\
\hline$A F A P 1-A S 1^{*}$ & AFAP1 antisense RNA 1 & 6.32 & 5.09 & 2.34 \\
\hline TRAF3 & Tnf receptor-associated factor 3 & 5.45 & 4.23 & 2.34 \\
\hline$N A V 3$ & Neuron navigator 3 & 6.9 & 5.68 & 2.34 \\
\hline GOS2 & G0/G1 switch 2 & 8.95 & 7.73 & 2.34 \\
\hline TMEM132A* & Transmembrane protein $132 \mathrm{~A}$ & 4.69 & 3.47 & 2.33 \\
\hline TMEM171* & Transmembrane protein 171 & 6.95 & 5.74 & 2.32 \\
\hline ICAM2 & Intercellular adhesion molecule 2 & 6.69 & 5.49 & 2.31 \\
\hline $\mathrm{ZC} 3 \mathrm{H} 12 \mathrm{C}$ & Zinc finger $\mathrm{CCCH}$-type containing $12 \mathrm{C}$ & 6.77 & 5.58 & 2.27 \\
\hline$M M P 9$ & Matrix metallopeptidase 9 & 3.54 & 2.38 & 2.24 \\
\hline
\end{tabular}


Table I. Continued

\begin{tabular}{|c|c|c|c|c|}
\hline \multirow[b]{2}{*}{ Gene symbol } & \multirow[b]{2}{*}{ Description } & \multicolumn{3}{|c|}{ Expression } \\
\hline & & $\mathrm{TNF} \alpha$ & Control & FC \\
\hline CXCL2 & Chemokine (C-X-C motif) ligand 2 & 4.04 & 2.89 & 2.23 \\
\hline$Z C 3 H 7 B$ & Zinc finger $\mathrm{CCCH}$-type containing $7 \mathrm{~B}$ & 5.92 & 4.77 & 2.22 \\
\hline STAP2* & Signal transducing adaptor family member 2 & 5.96 & 4.81 & 2.22 \\
\hline SERPINA1 & Serpin peptidase inhibitor, clade A (alpha- 1 antiproteinase, antitrypsin), member 1 & 2.98 & 1.85 & 2.19 \\
\hline$R H B D F 2 *$ & Rhomboid 5 homolog 2 (Drosophila) & 5.44 & 4.31 & 2.19 \\
\hline$C C L 2 *$ & Chemokine (C-C motif) ligand 2 & 2.9 & 1.77 & 2.19 \\
\hline NFKB1 & Nuclear factor of kappa light polypeptide gene enhancer in B-cells 1 & 6.69 & 5.57 & 2.18 \\
\hline$M L K L^{*}$ & Mixed lineage kinase domain-like & 6 & 4.89 & 2.16 \\
\hline LACC1 & Laccase (multicopper oxidoreductase) domain containing 1 & 6.35 & 5.24 & 2.16 \\
\hline$K D R^{*}$ & Kinase insert domain receptor & 4.98 & 3.88 & 2.14 \\
\hline$B M P E R^{*}$ & Bmp binding endothelial regulator & 4.41 & 3.32 & 2.14 \\
\hline LOC105369848 & Uncharacterized LOC105369848 & 3.21 & 2.11 & 2.14 \\
\hline IL23A & Interleukin 23 , alpha subunit p19 & 3.22 & 2.13 & 2.13 \\
\hline$P P I F$ & Peptidylprolyl isomerase $\mathrm{f}$ & 7.96 & 6.87 & 2.12 \\
\hline$L A M B 3$ & Laminin, beta 3 & 8.61 & 7.55 & 2.09 \\
\hline RLN3; IL27RA & Relaxin 3; interleukin 27 receptor, alpha & 5.35 & 4.29 & 2.09 \\
\hline CXCL1 & Chemokine (C-X-C motif) ligand 1 (melanoma growth stimulating activity, alpha) & 4.39 & 3.33 & 2.08 \\
\hline$P O U 2 F 2$ & Pou class 2 homeobox 2 & 3.82 & 2.78 & 2.06 \\
\hline KLHL36 & Kelch-like family member 36 & 4.37 & 3.36 & 2.02 \\
\hline MIR4454 & MicroRNA 4454 & 5.04 & 4.02 & 2.02 \\
\hline PDK4 & Pyruvate dehydrogenase kinase, isozyme 4 & 2.57 & 3.59 & -2.03 \\
\hline ITGB4 & Integrin beta 4 & 6.3 & 7.35 & -2.07 \\
\hline KLHLA & Kelch-like family member 4 & 5.61 & 6.7 & -2.12 \\
\hline GALNT5 & Polypeptide $n$-acetylgalactosaminyltransferase 5 & 4.72 & 5.8 & -2.13 \\
\hline SESN3 & Sestrin 3 & 2.59 & 3.69 & -2.14 \\
\hline$L G R 5$ & Leucine-rich repeat containing $\mathrm{G}$ protein-coupled receptor 5 & 2.11 & 3.27 & -2.25 \\
\hline RHOD & Ras homolog family member $d$ & 4.73 & 5.97 & -2.36 \\
\hline SLC $38 A 4$ & Solute carrier family 38 , member 4 & 3.46 & 4.81 & -2.54 \\
\hline VIPRI & Vasoactive intestinal peptide receptor 1 & 4.79 & 6.21 & -2.68 \\
\hline$S L C 2 A 12$ & Solute carrier family 2 (facilitated glucose transporter), member 12 & 3.06 & 4.77 & -3.26 \\
\hline
\end{tabular}

*Genes also significantly down-regulated when treated with $40 \mu \mathrm{M}$ apigenin. FC: Fold change relative to untreated controls; all FC had an ANOVA significance value of less than 0.05 .

chloroform method, the quality was assessed, and concentration was equalized to $82 \mathrm{ng} / \mu \mathrm{l}$ in nuclease-free water. Whole-transcriptome analysis was conducted according to the GeneChip ${ }^{\mathrm{TM}}$ WT PLUS Reagent Manual for Whole Transcript (WT) Expression Arrays (Affymetrix/Thermo Fisher Scientific Waltham, MA, USA). Briefly, RNA was synthesized to first-strand cDNA, second-strand cDNA and followed by transcription to cRNA. cRNA was purified and assessed for yield, before second cycle single-stranded cDNA synthesis, hydrolysis of RNA and purification of second cycle single-stranded cDNA. cDNA was then quantified for yield and equalized to $176 \mathrm{ng} / \mathrm{ml}$. Subsequently, cDNA was fragmented, labeled and hybridized onto the arrays before being subject to fluidics and imaging using the Gene Atlas (Affymetrix/Thermo Fisher Scientific). The array data quality control and initial processing from CEL to CHP files were conducted using expression console, before data evaluation using the Affymetrix transcriptome analysis console. Samples were run in triplicates $(n=3)$. The data have been deposited into the Gene Expression Omnibus for public analysis at https://www.ncbi.nlm.nih.gov/geo/query/acc.cgi?acc= GSE120550
Statistical analysis. Data analysis on microarrays was conducted using Affymetrix ${ }^{\circledR}$ Transcriptome Analysis Console (TAC) Software, with parameter set at: Array Type: HuGene-2_1-st, Genome Version: hg19 (Homo sapiens) Condition (Comparisons): API; Control; Cotx; TNF, gene-level fold change $<-2$ or $>2$, genelevel $p$-value $<0.05$, ANOVA Method: empirical Bayes statistics for differential expression. Other statistical analysis was performed using GraphPad Prism (version 3.0; GraphPad Software Inc. San Diego, CA, USA) with the significance of the difference between the groups assessed using a one-way ANOVA, followed by Tukey post-hoc comparison of means test.

\section{Results}

A non-lethal working concentration in MDA-MB-231 cells was established for $\mathrm{TNF} \alpha$, apigenin (as previously reported) (22) and IL1 $\alpha$ (Figure 1) where the sub-lethal working concentrations were set at the following: $40 \mu \mathrm{M}$ apigenin and $40 \mathrm{ng} / \mathrm{ml} \mathrm{TNF} \alpha$. To investigate TNF $\alpha$-mediated induction of 


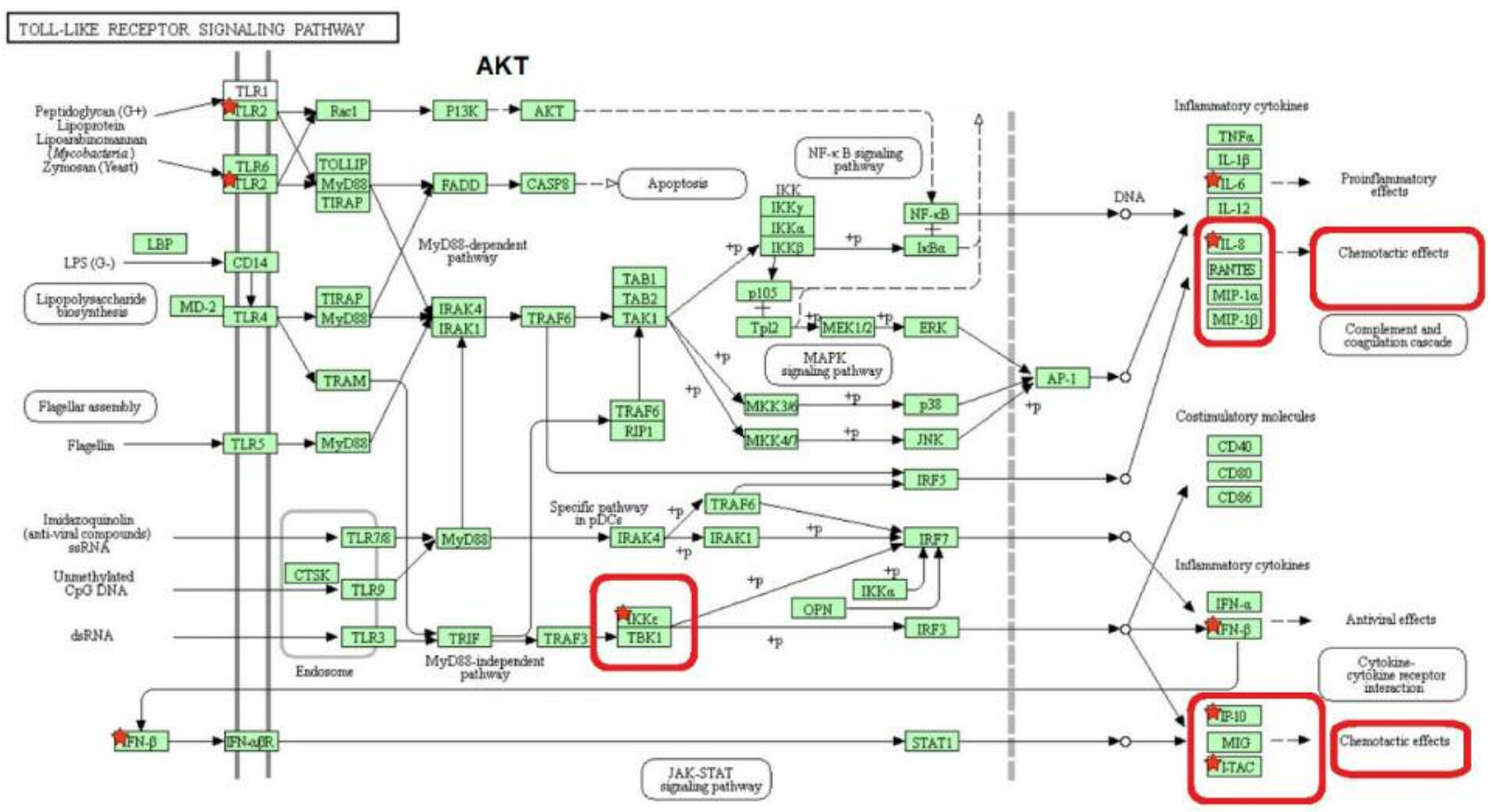

Figure 3. Differential whole transcriptome assay where stars denote genes up-regulated by tumor necrosis factor- $\alpha(T N F \alpha)(40 \mathrm{ng} / \mathrm{ml})$ and downregulated by apigenin $(40 \mu M)$. Official gene symbols were uploaded from the Database for Annotation, Visualization and Integrated Discovery (DAVID) v6.8 where annotation files representing pathways show significant changes to the KEGG pathway for Toll-like receptor pathways.

Table II. Biological process analysis: Comparing controls vs. tumor necrosis factor- $\alpha$ (TNF $\alpha)$-treated MDA-MB-231 cells. Up-regulated genes were imported into Gene Ontology Consortium at geneontology.org using the Panther Classification system enrichment analysis by Go-Slim Biological Process. Classifications identified show TNFa largely affected cytokine-mediated signaling pathways (A), being further defined as primarily involved with immune cell migration and regulation of angiogenesis $(B)$.

Analysis type: PANTHER overrepresentation test

Annotation version and release date: Go Ontology database released 2018-08-09

Analyzed list: Up-regulated by TNFa vs. control

Reference list: Homo sapiens (all genes in database)

Annotation data set: Go Biological process complete

Test type: Fisher's exact with false discovery rate (FDR) multiple correction

Displaying only results with an FDR $<0.05$

Reference list

21,042 out of 21,042

Mapped IDs:

Unmapped IDs:

Multiple Mapping information
0
Uploaded data set

66 out of 68

9

2

A. PANTHER GO-Slim Biological Process Homo sapiens

(REF)

Cytokine - mediated signaling pathway

60

B. Go Biological Process Complete

Homo sapiens

$\#$

(REF)

Fold

enrichment

FDR

T cell extravasation (leukocyte migration, motility)

( $\mathrm{T}$ cell/lymphocyte migration)

\begin{tabular}{rrrccc}
351 & 10 & 1.17 & 8.57 & $2.93 \mathrm{E}-07$ & $3.46 \mathrm{E}-05$ \\
21 & 4 & 0.07 & 58.94 & $1.18 \mathrm{E}-06$ & $3.58 \mathrm{E}-03$ \\
1407 & 20 & 4.55 & 4.4 & $1.24 \mathrm{E}-08$ & $2.06 \mathrm{E}-06$ \\
282 & 10 & 0.91 & 10.97 & $3.15 \mathrm{E}-08$ & $4.77 \mathrm{E}-06$ \\
\hline
\end{tabular}

Regulation of vascular would healing (response to stress)

Regulation of angiogenesis

0.91

4.4

$3.15 \mathrm{E}-08$

$.77 \mathrm{E}-06$ 


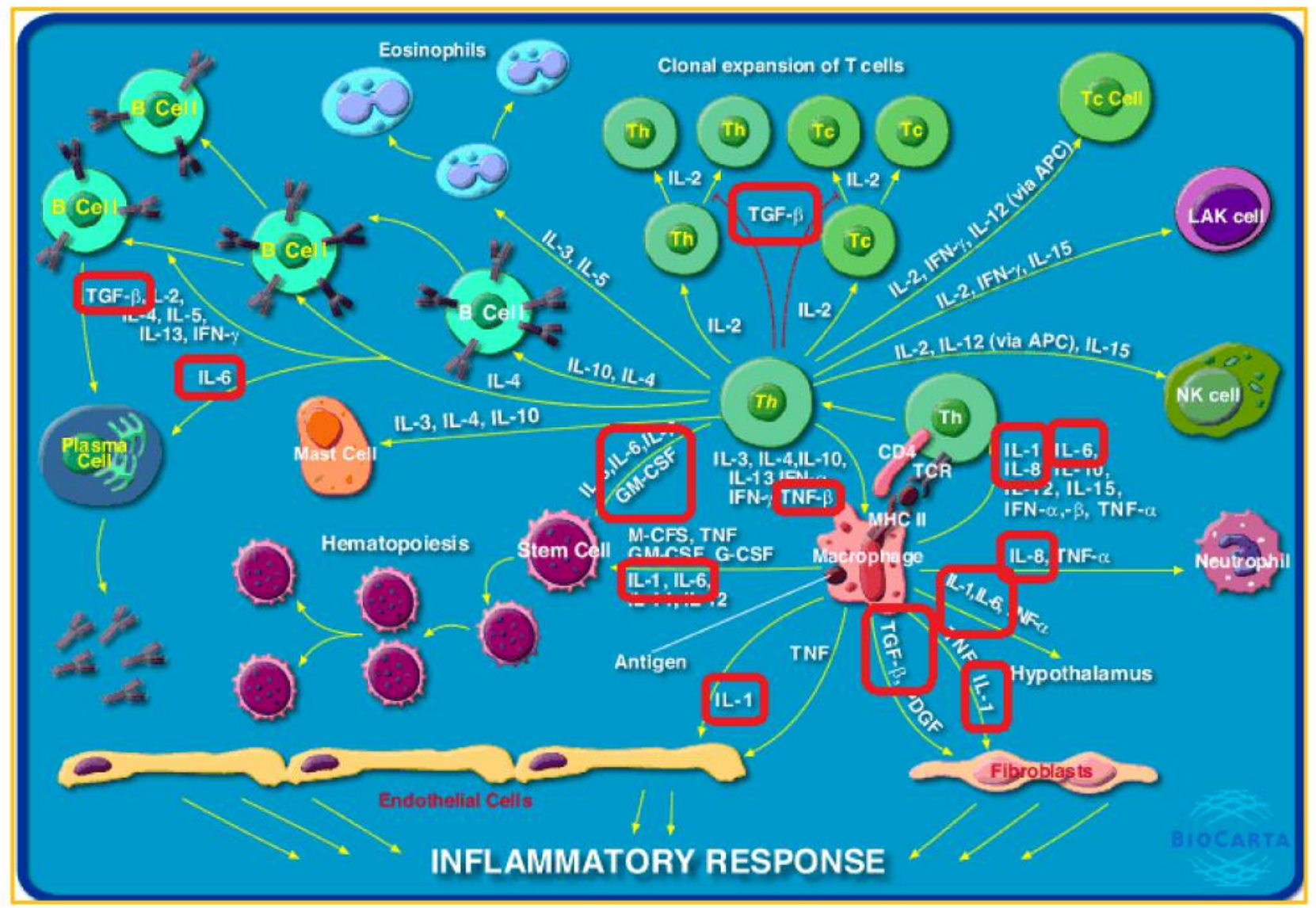

Figure 4. Differential whole transcriptome assay highlighting genes in MDA-MB-231 cells up-regulated by tumor necrosis factor- $\alpha$ (TNF $\alpha$; $40 \mathrm{ng} / \mathrm{ml})$ and down-regulated by apigenin $(40 \mu \mathrm{M})$. Official gene symbols were uploaded from the Database for Annotation, Visualization and Integrated Discovery (DAVID) v6.8 where annotation files representing pathways show significant changes to the Biocarta Inflammatory response pathway. Th: T-Helper cells; LAK: lymphokine-activated killer cell; Tc: T-cytotoxic cells; IL: interleukin; TGF: transforming growth factor; IFN: interferon; PDGF: platelet-derived growth factor; APC: antigen-presenting cell; NK: natural killer.

chemokines and the influence of apigenin, whole transcriptomic microarray analysis was conducted using the Affymetrix gene atlas system. The differences between TNF $\alpha$ and the untreated controls are shown by volcano plot (Figure 2) and listed in Table I. The data confirm a TNF $\alpha$ evoked a rise in ILIA, IKBKE, IL6, CCL2 and CSF2, as previously reported (22); in addition 75 genes were upregulated and 10 down-regulated. Using GeneOntology, Panther Classification Systems, Annotation dataset, Panther Go-Slim biological process, the results show cytokinemediated signaling to be the primary processes affected by the presence of TNF $\alpha$, with a false-discovery rate value of less than 0.05 (Table II) and the top two events affected by TNF $\alpha$ being T-cell extravasation (leukocyte migration: T-cell migration, lymphocyte migration), and vascularization (regulation of angiogenesis, and vascular development) (Table II).
While the differences between the effects of TNF $\alpha$ and TNF $\alpha$ combined with apigenin were vast, many effects were under the control of apigenin alone. The interfering variables were eliminated, and analysis was performed for transcriptional changes due to TNF $\alpha$ alone, and which were directly influenced by the presence of apigenin (Table III). The results corroborate our previous work in showing IKBKE to be a central controlling transcription factor as demonstrated in a Kegg overlay map (Figure 3) and Biocarta inflammatory scheme (Figure 4). Given that altered expression of ILIA was a primary outcome of TNF $\alpha$ treatment, we then evaluated whether IL $1 \alpha$ alone was able to elicit the same effect in up-regulating release of CCL2. The data confirm this to be the case (Figure 5A); both effects were reduced by apigenin with correlating changes for the $I K B K E$ transcript (Figure 5B). These findings show that TNF $\alpha$ can up-regulate $I L 1 A$, which is likely to perpetuate a 
Table III. Differential whole transcriptome changes: Genes up-regulated by $40 \mathrm{ng} / \mathrm{ml}$ tumor necrosis factor- $\alpha(T N F \alpha)$ and which were downregulated by $40 \mu M$ apigenin in $M D A-M B-231$ cells. The data are fold change (FC) in expression. *See Table I for full gene names.

\begin{tabular}{|c|c|c|c|c|c|}
\hline \multirow[b]{2}{*}{ Gene* } & \multicolumn{2}{|c|}{$\mathrm{TNF} \alpha$} & \multicolumn{2}{|c|}{ TNF $\alpha+$ apigenin } & \multirow{2}{*}{$\begin{array}{c}\text { Effect of } \\
\text { apigenin } \\
\text { reduction } \\
\text { in expression }\end{array}$} \\
\hline & FC & $p$-Value & $\mathrm{FC}$ & $p$-Value & \\
\hline$I L 1 A$ & 21.57 & $<0.001$ & -15.16 & $<0.001$ & $70 \%$ \\
\hline CXCL8 & 20.61 & $<0.001$ & -2.22 & $<0.001$ & $11 \%$ \\
\hline$C S F 2$ & 6.04 & $<0.001$ & -2.36 & 0.007 & $39 \%$ \\
\hline CTSS & 5.97 & 0.001 & -3.96 & $<0.001$ & $66 \%$ \\
\hline ADAMTS9 & 5.69 & $<0.001$ & -2.11 & $<0.001$ & $37 \%$ \\
\hline$C 3$ & 4.92 & $<0.001$ & -4.5 & $<0.001$ & $91 \%$ \\
\hline$I K B K E$ & 4.55 & $<0.001$ & -4.92 & $<0.001$ & $108 \%$ \\
\hline$L A M C 2$ & 4.48 & $<0.001$ & -3.2 & $<0.001$ & $71 \%$ \\
\hline$T L R 2$ & 3.73 & $<0.001$ & -2.93 & $<0.001$ & $79 \%$ \\
\hline GPRC $5 B$ & 3.66 & 0.001 & -3.44 & 0.001 & $94 \%$ \\
\hline CNTNAP1 & 3.6 & 0.002 & -2.45 & $<0.001$ & $68 \%$ \\
\hline CLDN1 & 3.6 & $<0.001$ & -2.53 & $<0.001$ & $70 \%$ \\
\hline IL6 & 3.25 & 0.020 & -2.85 & 0.043 & $88 \%$ \\
\hline SERP 2 ; SERPB 10 & 3.01 & $<0.001$ & -3.51 & $<0.001$ & $117 \%$ \\
\hline NFATC2 & 3.01 & 0.033 & -4.02 & 0.009 & $134 \%$ \\
\hline CXCL10 & 2.94 & 0.007 & -2.45 & 0.010 & $83 \%$ \\
\hline CXCL11 & 2.93 & 0.001 & -3.46 & $<0.001$ & $118 \%$ \\
\hline IRAK3 & 2.73 & 0.017 & -2.53 & 0.005 & $93 \%$ \\
\hline$N R 3 C 2$ & 2.68 & 0.033 & -3.68 & 0.010 & $137 \%$ \\
\hline IL32 & 2.63 & 0.002 & -2.34 & 0.002 & $89 \%$ \\
\hline SERPINE1 & 2.61 & $<0.001$ & -2.02 & 0.002 & $77 \%$ \\
\hline$I L 24$ & 2.49 & 0.003 & -4.84 & $<0.001$ & $194 \%$ \\
\hline DRAMI & 2.44 & 0.032 & -2.19 & 0.035 & $90 \%$ \\
\hline TNFSF 15 & 2.41 & 0.003 & -2.49 & 0.006 & $103 \%$ \\
\hline SLIT2 & 2.39 & 0.004 & -3 & 0.002 & $126 \%$ \\
\hline$A F A P 1-A S 1$ & 2.34 & 0.035 & -2.33 & 0.030 & $100 \%$ \\
\hline TMEM132A & 2.33 & 0.003 & -2.1 & $<0.001$ & $90 \%$ \\
\hline TMEM171 & 2.32 & 0.001 & -2.04 & 0.004 & $88 \%$ \\
\hline STAP2 & 2.22 & 0.001 & -2.22 & $<0.001$ & $100 \%$ \\
\hline CCL2 & 2.19 & 0.002 & -2.12 & 0.003 & $97 \%$ \\
\hline$R H B D F 2$ & 2.19 & 0.003 & -2.07 & $<0.001$ & $95 \%$ \\
\hline$M L K L$ & 2.16 & 0.001 & -2.3 & 0.001 & $106 \%$ \\
\hline$K D R$ & 2.14 & 0.032 & -3.09 & 0.003 & $144 \%$ \\
\hline BMPER & 2.14 & 0.008 & -3.02 & 0.001 & $141 \%$ \\
\hline KLHL36 & 2.02 & 0.048 & -2.66 & 0.005 & $132 \%$ \\
\hline
\end{tabular}

sustained release of CCL2, both of which can be downregulated by apigenin.

\section{Discussion}

The data in this study show that $I L 1 A$ was the transcript most highly up-regulated by TNF $\alpha$ (by 21.57-fold, $p<0.001$ ), which was suppressed by apigenin (by 15.16-fold, $p<0.001$ ) in MDA-MB-231 cells. TNF $\alpha$ along with IL1 $\alpha$, and corresponding cognate receptors are ubiquitous in the tumor milieu of highly invasive carcinomas (25) and correspond to
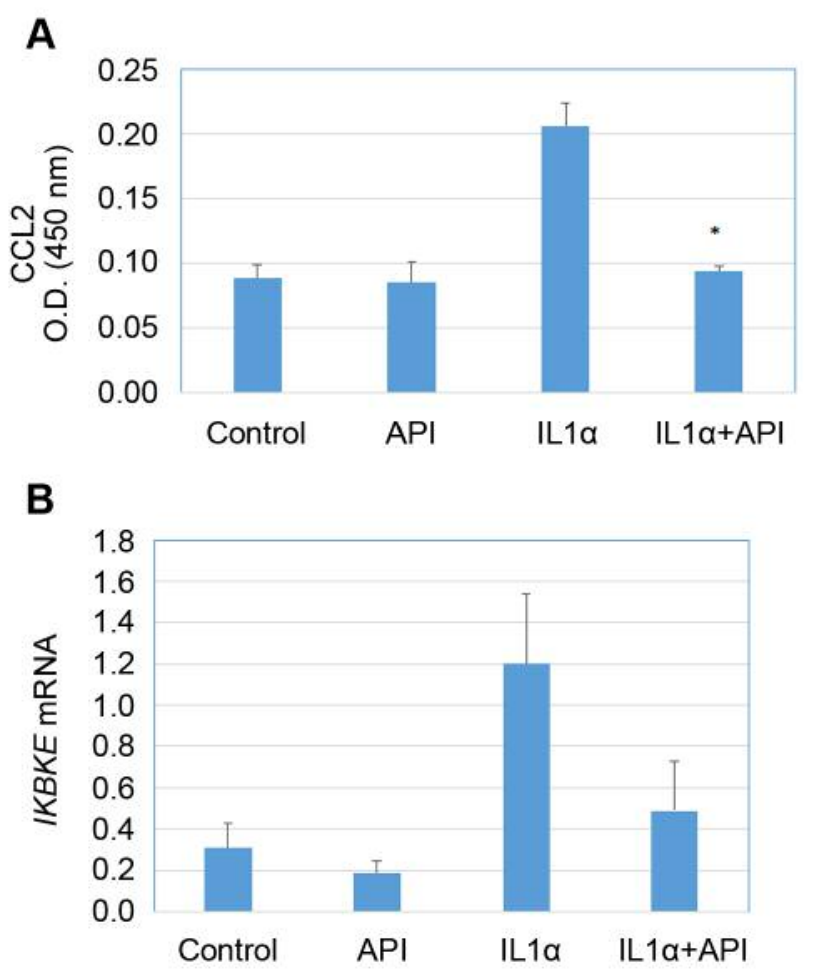

Figure 5. A: Enzyme-linked immunosorbent assays were used to determine the effect of apigenin (API) on interleukin 1-alpha (ILI $\alpha)$-induced and tumor necrosis factor alpha (TNF $\alpha)$ changes in $C$ - $C$ motif chemokine ligand 2 (CCL2) release by $M D A-M B-231$ cells. The data represent CCL2 concentration $(\mathrm{pg} / \mathrm{ml})$ and are presented as the mean \pm S.E.M. $(n=3) . B$ : Polymerase chain reaction analysis of inhibitor of nuclear factor kappa$B$ kinase subunit epsilon (IKBKE) transcription in MDA-MB-231 cells with/without IL1a/API. The data represent normalized expression and are expressed as the mean \pm S.E.M. $(n=3$.) The significance of differences between the control and treated groups was determined by one-way ANOVA using the Tukey post-hoc test. *Significant differences between (ILI $\alpha$ vs. ILI $\alpha+$ API) groups at $p<0.05$.

larger tumor size, lymph node metastasis and overall poor prognosis $(26,27)$ and overexpression of certain chemokines (e.g. CCL2, IL8 and IL6). All of these cytokines were suppressed by apigenin in the presence of $\mathrm{TNF} \alpha$ as demonstrated in this study. The capacity of apigenin to attenuate all of these pro-oncogenic processes simultaneously would, in theory, hamper infiltration of CCR2/CCR5 receptor-bearing LSPs or myeloid cells into the tumor milieu (28), which on their own amplify expression of tumorpromoting cytokines, growth/motility factor, and tumor evasion tactics (29-35).

While the data in this work show that apigenin can reduce a diverse range of $\mathrm{TNF} \alpha$-induced, tumor-promoting transcripts, often associated with tumor invasion, there was a near complete reduction of complement component $\mathrm{C} 3$, the 
relevance of which needs further investigation. C3 is involved in the innate immune response, but high concentrations are found in the serum of patients with breast and lung cancer also considered a plausible diagnostic biomarker (36-39). The C3 convertase complex and initial cleavage of the C3 molecule activates the complement system, where downstream products ( $\mathrm{C} 3 \mathrm{a}$ and $\mathrm{C} 3 \mathrm{~b})$ are deposited around solid tumors concomitant to overexpression of surfaceexpressed CD proteins that protect cells from complementdependent cytotoxicity. Overexpressed C3s correspond to immune evasion (40) loss of dendritic cell-mediated crosspresentation (41) and antibody-dependent cell-mediated cytotoxicity (42). In contrast, chemotherapy drugs that reactivate the complement system-evoked cell death (43-45) and antibody-dependent cell-mediated cytotoxicity (46) or directly target C3 are considered a means of reactivating tumor immunosurveillance (47). There is a need to further investigate the role of apigenin in this process, as reducing C3 confers a protective effect against carcinogenesis.

In addition to $C 3, C C L 2, I L 8, I L 6$ and $I L 1 A$, the data in this study support the notion that apigenin can reduce expression of numerous other oncogenes which drive metastatic tumor growth such as cathepsin S (CS) and laminin subunit gamma 2 (LAMC2) (48-52). These findings, as a whole, add to the existing body of literature demonstrating diverse anticancer effects for apigenin (27) reported to reduce tumor growth (53), migration, invasion, metastasis, and concomitant signaling targets [e.g. extracellular signal-regulated kinase, c-Jun $N$-terminal kinase activator protein 1, nuclear factor kappa-light-chain-enhancer of activated $\mathrm{B}$ cells $(54,55)$, mitogen-activated protein kinase, PI3K/protein kinase B (56), glycogen synthase kinase-3 beta (57), Janus kinases/signal transducer and activator of transcription proteins and WNT/beta-catenin pathways (58)]. Moreover, apigenin can also master expression of anticancer microRNAS such as $m i R-520 b$ and miR-101 (59) while sensitizing to the effects of diverse chemotherapy drugs (60) and overcoming resistance (58).

In summary, in this study we present a global view of the anticancer/anti-inflammatory properties of apigenin specific to the influence of TNF $\alpha$ in a TNBC cell model (Public Data set Gene Expression Omnibus file https://www.ncbi.nlm.nih.gov/ geo/query/acc.cgi?acc=GSE120550).

\section{Conflicts of Interest}

The Authors declare that they have no conflict of interest in regard to this study.

\section{Authors' Contributions}

DB conducted the primary research, including all bench work studies pertaining to apigenin and $\mathrm{TNF} \alpha$ in addition to article preparation. EM carried out the transcriptomic microarray study, including all quality control steps in addition to article preparation. KS provided oversight management including consultation, research direction in addition to the preparation of the article. All listed Authors declare consent to the article's publication.

\section{Acknowledgements}

This research was supported by the National Institute of Minority Health and Health Disparities of the National Institutes of Health through Grant Number U54 MD007582 and Grant Number P20 MD006738

\section{References}

1 Newman LA and Kaljee LM: Health disparities and triplenegative breast cancer in African American women: A review. JAMA Surg 152(5): 485-493, 2017. PMID: 28355428. DOI: 10.1001/jamasurg.2017.0005

2 Gerratana L, Basile D, Buono G, De Placido S, Giuliano M, Minichillo S, Coinu A, Martorana F, De Santo I, Del Mastro L, De Laurentiis M, Puglisi F and Arpino G: Androgen receptor in triple-negative breast cancer: A potential target for the targetless subtype. Cancer Treat Rev 68: 102-110, 2018. PMID: 29940524. DOI: $10.1016 /$ j.ctrv.2018.06.005

3 Sharma P: Update on the treatment of early-stage triple-negative breast cancer. Curr Treat Options Oncol 19(5): 22, 2018. PMID: 29656345. DOI: $10.1007 / \mathrm{s} 11864-018-0539-8$

4 Terret C and Russo C: Pharmacotherapeutic management of breast cancer in elderly patients: The promise of novel agents. Drugs Aging 35(2): 93-115, 2018. PMID: 29388072. DOI: 10.1007/s40266-018-0519-5

5 Shah N, Mohammad AS, Saralkar P, Sprowls SA, Vickers SD, John D, Tallman RM, Lucke-Wold BP, Jarrell KE, Pinti M, Nolan RL and Lockman PR: Investigational chemotherapy and novel pharmacokinetic mechanisms for the treatment of breast cancer brain metastases. Pharmacol Res 132: 47-68, 2018. PMID: 29604436. DOI: 10.1016/j.phrs.2018.03.021

6 Tong CWS, Wu M, Cho WCS and To KKW: Recent advances in the treatment of breast cancer. Front Oncol 8: 227, 2018. PMID: 29963498. DOI: 10.3389/fonc.2018.00227

7 Whiteside TL and Herberman RB: The role of natural killer cells in immune surveillance of cancer. Curr Opin Immunol 7(5): 704710, 1995. PMID: 8573315.

8 Wu JD, Higgins LM, Steinle A, Cosman D, Haugk K and Plymate SR: Prevalent expression of the immunostimulatory MHC class I chain-related molecule is counteracted by shedding in prostate cancer. J Clin Invest 114(4): 560-568, 2004. PMID: 15314693. DOI: $10.1172 / J C I 22206$

9 Yoon SR, Chung JW and Choi I: Development of natural killer cells from hematopoietic stem cells. Mol Cells 24(1): 1-8, 2007. PMID: 17846493.

10 Marzbani E, Inatsuka C, Lu H and Disis ML: The invisible arm of immunity in common cancer chemoprevention agents. Cancer Prev Res 6(8): 764-773, 2013. PMID: 23918793. DOI: 10.1158/ 1940-6207.CAPR-13-0036

11 Li Z, Qiu Y, Lu W, Jiang Y and Wang J: Immunotherapeutic interventions of triple-negative breast cancer. J Transl Med 16(1): 147, 2018. PMID: 29848327. DOI: 10.1186/s12967-018$1514-7$ 
12 Santoni M, Romagnoli E, Saladino T, Foghini L, Guarino S Capponi M, Giannini M, Cognigni PD, Ferrara G and Battelli $\mathrm{N}$ : Triple-negative breast cancer: Key role of tumor-associated macrophages in regulating the activity of anti-PD-1/PD-L1 agents. Biochim Biophys Acta Rev Cancer 1869(1): 78-84, 2018. PMID: 29126881. DOI: 10.1016/j.bbcan.2017.10.007

13 Tolba MF and Omar HA: Immunotherapy, an evolving approach for the management of triple-negative breast cancer: Converting non-responders to responders. Crit Rev Oncol Hematol 122: 202-207, 2018. PMID: 29373180. DOI: 10.1016/j.critrevonc. 2018.01.005

14 Tan TJ, Chan JJ, Kamis S and Dent RA: What is the role of immunotherapy in breast cancer? Chin Clin Oncol 7(2): 13, 2018. PMID: 29764158. DOI: 10.21037/cco.2018.04.01

15 Polk A, Svane IM, Andersson M and Nielsen D: Checkpoint inhibitors in breast cancer-current status. Cancer Treat Rev 63: 122-134, 2018. PMID: 29287242. DOI: 10.1016/j.ctrv.2017.12.008

16 Monneur A, Goncalves A and Bertucci F: PD-L1 expression and PD-1/PD-L1 inhibitors in breast cancer. Bull Cancer 105(3): 263274, 2018. PMID: 29455872. DOI: 10.1016/j.bulcan.2017.11.012

$17 \mathrm{Kwa}$ MJ and Adams S: Checkpoint inhibitors in triplenegative breast cancer (TNBC): Where to go from here. Cancer 124(10): 2086-2103, 2018. PMID: 29424936. DOI: $10.1002 / \mathrm{cncr} .31272$

18 Kim JM, Noh EM, Song HK, You YO, Jung SH, Kim JS, Kwon $\mathrm{KB}$, Lee YR and Youn HJ: Silencing of casein kinase 2 inhibits pkc-induced cell invasion by targeting MMP9 in MCF7 cells. Mol Med Rep 17(6): 8397-8402, 2018. PMID: 29658601. DOI: 10.3892/mmr.2018.8885

19 Hong J, Fristiohady A, Nguyen CH, Milovanovic D, Huttary N, Krieger S, Hong J, Geleff S, Birner P, Jager W, Ozmen A, Krenn $\mathrm{L}$ and Krupitza G: Apigenin and luteolin attenuate the breaching of MDA-MB231 breast cancer spheroids through the lymph endothelial barrier in vitro. Front Pharmacol 9: 220, 2018. PMID: 29593542. DOI: 10.3389/fphar.2018.00220

20 Bensasson RV, Zoete V, Jossang A, Bodo B, Arimondo PB and Land EJ: Potency of inhibition of human DNA topoisomerase I by flavones assessed through physicochemical parameters. Free Radic Biol Med 51(7): 1406-1410, 2011. PMID: 21745563. DOI: 10.1016/j.freeradbiomed.2011.06.021

21 Ye Q, Kantonen S and Gomez-Cambronero J: Serum deprivation confers the MDA-MB-231 breast cancer line with an EGFR/JAK3/PLD2 System that maximizes cancer cell invasion. J Mol Biol 425(4): 755-766, 2013. PMID: 23238254. DOI: 10.1016/j.jmb.2012.11.035

22 Bauer D, Redmon N, Mazzio E and Soliman KF: Apigenin inhibits TNFalpha/IL-1alpha-induced CCL2 release through IKBK-epsilon signaling in MDA-MB-231 human breast cancer cells. Plos One 12(4): e0175558, 2017. PMID: 28441391. DOI: 10.1371/journal.pone. 0175558

23 Seo HS, Ku JM, Choi HS, Woo JK, Lee BH, Kim DS, Song HJ, Jang BH, Shin YC and Ko SG: Apigenin overcomes drug resistance by blocking the signal transducer and activator of transcription 3 signaling in breast cancer cells. Oncol Rep 38(2): 715-724, 2017. PMID: 28656316. DOI: 10.3892/or.2017.5752

24 Wesolowska O, Hendrich AB, Laniapietrzak B, Wisniewski J, Molnar J, Ocsovszki I and Michalak K: Perturbation of the lipid phase of a membrane is not involved in the modulation of MRP1 transport activity by flavonoids. Cell Mol Biol Lett 14(2): 199221, 2009. PMID: 19020811. DOI: 10.2478/s11658-008-0044-3
25 Kurtzman SH, Anderson KH, Wang Y, Miller LJ, Renna M, Stankus M, Lindquist RR, Barrows G and Kreutzer DL: Cytokines in human breast cancer: IL-1alpha and Il-1beta expression. Oncol Rep 6(1): 65-70, 1999. PMID: 9864403.

26 Sawai H, Takeyama H, Yamamoto M, Furuta A, Funahashi H, Okada Y, Sato M, Tanaka M and Manabe T: Enhancement of integrins by interleukin-1alpha, and their relationship with metastatic and invasive behavior of human pancreatic ductal adenocarcinoma cells. J Surg Oncol 82(1): 51-56, 2003. PMID: 12501168. DOI: $10.1002 /$ jso. 10187

27 Singer CF, Hudelist G, Gschwantler-Kaulich D, Fink-Retter A, Mueller R, Walter I, Czerwenka K and Kubista E: Interleukin1alpha protein secretion in breast cancer is associated with poor differentiation and estrogen receptor alpha negativity. Int $\mathbf{J}$ Gynecol Cancer 16(Suppl 2): 556-559, 2006. PMID: 17010072. DOI: $10.1111 /$ j.1525-1438.2006.00695.x

28 Kumar S, Kishimoto H, Chua HL, Badve S, Miller KD, Bigsby RM and Nakshatri H: Interleukin-1 alpha promotes tumor growth and cachexia in MCF-7 xenograft model of breast cancer. Am J Pathol 163(6): 2531-2541, 2003. PMID: 14633625.

29 Kuan EL and Ziegler SF: A tumor-myeloid cell axis, mediated via the cytokines IL-1alpha and TSLP, promotes the progression of breast cancer. Nat Immunol 19(4): 366-374, 2018. PMID: 29556001. DOI: 10.1038/s41590-018-0066-6

30 Nozaki S, Sledge GW, Jr. and Nakshatri H: Cancer cell-derived interleukin 1alpha contributes to autocrine and paracrine induction of pro-metastatic genes in breast cancer. Biochem Biophys Res Commun 275(1): 60-62, 2000. PMID: 10944441. DOI: $10.1006 /$ bbrc.2000.3241

31 Hutchins D and Steel CM: Regulation of ICAM-1 (CD54) expression in human breast cancer cell lines by interleukin 6 and fibroblast-derived factors. Int J Cancer 58(1): 80-84, 1994. PMID: 7516927.

32 Verhasselt B, Van Damme J, van Larebeke N, Put W, Bracke M, De Potter C and Mareel M: Interleukin-1 is a motility factor for human breast carcinoma cells in vitro: Additive effect with interleukin-6. Eur J Cell Biol 59(2): 449-457, 1992. PMID: 1493810 .

33 Bhat-Nakshatri P, Newton TR, Goulet R Jr. and Nakshatri H: NF-kappab activation and interleukin 6 production in fibroblasts by estrogen receptor-negative breast cancer cell-derived interleukin 1alpha. Proc Natl Acad Sci USA 95(12): 6971-6976, 1998. PMID: 9618523.

34 Sedlak J, Speiser P, Hunakova L, Duraj J and Chorvath B: Modulation of EGF receptor and CD15 (LewisX) antigen on the cell surface of breast carcinoma cell lines induced by cytokines, retinoic acid, 12-O-tetradecanoylphorbol 13-acetate and 1,25(OH)2-vitamin D3. Chemotherapy 40(1): 51-56, 1994. PMID: 7905817. DOI: 10.1159/000239170

35 Uria JA, Stahle-Backdahl M, Seiki M, Fueyo A and Lopez-Otin $\mathrm{C}$ : Regulation of collagenase-3 expression in human breast carcinomas is mediated by stromal-epithelial cell interactions. Cancer Res 57(21): 4882-4888, 1997. PMID: 9354453.

36 Opstal-van Winden AW, Vermeulen RC, Peeters PH, Beijnen JH and van Gils $\mathrm{CH}$ : Early diagnostic protein biomarkers for breast cancer: How far have we come? Breast Cancer Res Treat 134(1): 1-12, 2012. PMID: 22179926. DOI: 10.1007/s10549-011-1907-2

37 Michlmayr A, Bachleitner-Hofmann T, Baumann S, MarchettiDeschmann M, Rech-Weichselbraun I, Burghuber C, Pluschnig U, Bartsch R, Graf A, Greil R, Allmaier G, Steger G, Gnant M, 
Bergmann M and Oehler R: Modulation of plasma complement by the initial dose of epirubicin/docetaxel therapy in breast cancer and its predictive value. Br J Cancer 103(8): 1201-1208, 2010. PMID: 20877360. DOI: 10.1038/sj.bjc.6605909

38 Fan Y, Wang J, Yang Y, Liu Q, Fan Y, Yu J, Zheng S, Li M and Wang J: Detection and identification of potential biomarkers of breast cancer. J Cancer Res Clin Oncol 136(8): 1243-1254, 2010. PMID: 20237941. DOI: 10.1007/s00432-010-0775-1

39 Solassol J, Rouanet P, Lamy PJ, Allal C, Favre G, Maudelonde $\mathrm{T}$ and Mange A: Serum protein signature may improve detection of ductal carcinoma in situ of the breast. Oncogene 29(4): 550560, 2010. PMID: 19855429. DOI: 10.1038/onc.2009.341

40 Reis ES, Mastellos DC, Ricklin D, Mantovani A and Lambris JD: Complement in cancer: Untangling an intricate relationship. Nat Rev Immunol 18(1): 5-18, 2018. PMID: 28920587. DOI: 10.1038/nri.2017.97

41 Ren Z, Guo J, Liao J, Luan Y, Liu Z, Sun Z, Liu X, Liang Y, Peng $\mathrm{H}$ and Fu YX: Ctla-4 limits anti-CD20-mediated tumor regression. Clin Cancer Res 23(1): 193-203, 2017. PMID: 27354469. DOI: 10.1158/1078-0432.CCR-16-0040

42 Fishelson Z, Donin N, Zell S, Schultz S and Kirschfink M: Obstacles to cancer immunotherapy: Expression of membrane complement regulatory proteins (MCRPS) in tumors. Mol Immunol 40(2-4): 109-123, 2003. PMID: 12914817.

43 Lindorfer MA, Cook EM, Tupitza JC, Zent CS, Burack R, de Jong RN, Beurskens FJ, Schuurman J, Parren PW and Taylor RP: Real-time analysis of the detailed sequence of cellular events in mAb-mediated complement-dependent cytotoxicity of B-cell lines and of chronic lymphocytic leukemia B-cells. Mol Immunol 70: 13-23, 2016. PMID: 26690706. DOI: 10.1016/ j.molimm.2015.12.007

44 Vacchelli E, Pol J, Bloy N, Eggermont A, Cremer I, Fridman WH, Galon J, Marabelle A, Kohrt H, Zitvogel L, Kroemer G and Galluzzi L: Trial watch: Tumor-targeting monoclonal antibodies for oncological indications. Oncoimmunology 4(1): e985940, 2015. PMID: 25949870. DOI: 10.4161/2162402X.2014.985940

45 Pierpont TM, Limper CB and Richards KL: Past, present, and future of rituximab-the world's first oncology monoclonal antibody therapy. Front Oncol 8: 163, 2018. PMID: 29915719. DOI: $10.3389 /$ fonc. 2018.00163

46 Golay J: Direct targeting of cancer cells with antibodies: What can we learn from the successes and failure of unconjugated antibodies for lymphoid neoplasias? J Autoimmun 85: 6-19, 2017. PMID: 28666691. DOI: 10.1016/j.jaut.2017.06.002

47 Zha H, Wang X, Zhu Y, Chen D, Han X, Yang F, Gao J, Hu C, Shu C, Feng Y, Tan Y, Zhang J, Li Y, Wan YY, Guo B and Zhu B: Intracellular activation of complement C3 leads to PD-L1 antibody treatment resistance by modulating tumor-associated macrophages. Cancer Immunol Res 7(2): 193-207, 2019. PMID: 30514794. DOI: $10.1158 / 2326-6066 . C I R-18-0272$

48 Lin Y, Ge X, Zhang X, Wu Z, Liu K, Lin F, Dai C, Guo W and Li J: Protocadherin-8 promotes invasion and metastasis via laminin subunit gamma2 in gastric cancer. Cancer Sci 109(3): 732-740, 2018. PMID: 29325230. DOI: 10.1111/cas.13502

49 Ding J, Yang C and Yang S: Linc00511 interacts with mir-765 and modulates tongue squamous cell carcinoma progression by targeting LAMC2. J Oral Pathol Med 47(5): 468-476, 2018. PMID: 29315846. DOI: 10.1111/jop.12677

50 Rao G, Pierobon M, Kim IK, Hsu WH, Deng J, Moon YW, Petricoin EF, Zhang YW, Wang Y and Giaccone G: Inhibition of
AKT1 signaling promotes invasion and metastasis of non-small cell lung cancer cells with $K$-RAS or EGFR mutations. Sci Rep 7(1): 7066, 2017. PMID: 28765579. DOI: 10.1038/s41598-01706128-9

51 Huang D, Du C, Ji D, Xi J and Gu J: Overexpression of LAMC2 predicts poor prognosis in colorectal cancer patients and promotes cancer cell proliferation, migration, and invasion. Tumour Biol 39(6): 1010428317705849, 2017. PMID: 28653 882. DOI: $10.1177 / 1010428317705849$

52 Shen S, Gui T and Ma C: Identification of molecular biomarkers for pancreatic cancer with MRMR shortest path method. Oncotarget 8(25): 41432-41439, 2017. PMID: 28611293. DOI: 10.18632/oncotarget.18186

53 Yang J, Pi C and Wang G: Inhibition of PI3K/AKT/MTOR pathway by apigenin induces apoptosis and autophagy in hepatocellular carcinoma cells. Biomed Pharmacother 103: 699707, 2018. PMID: 29680738. DOI: 10.1016/j.biopha. 2018.04.072

$54 \mathrm{Wu}$ TC, Chan ST, Chang CN, Yu PS, Chuang CH and Yeh SL: Quercetin and chrysin inhibit nickel-induced invasion and migration by downregulation of TLR4/NF-kappab signaling in A549 cells. Chem Biol Interact 292: 101-109, 2018. PMID: 30016632. DOI: 10.1016/j.cbi.2018.07.010

55 Xia Y, Yuan M, Li S, Thuan UT, Nguyen TT, Kang TW, Liao W, Lian S and Jung YD: Apigenin suppresses the IL-1beta-induced expression of the urokinase-type plasminogen activator receptor by inhibiting MAPK-mediated AP-1 and NF-kappab signaling in human bladder cancer T24 cells. J Agric Food Chem 66(29): 7663-7673, 2018. PMID: 29945448. DOI: 10.1021/acs.jafc. $8 \mathrm{~b} 02351$

56 Madunic J, Madunic IV, Gajski G, Popic J and Garaj-Vrhovac V: Apigenin: A dietary flavonoid with diverse anticancer properties. Cancer Lett 413: 11-22, 2018. PMID: 29097249. DOI: $10.1016 /$ j.canlet.2017.10.041

57 Zhou Z, Tang M, Liu Y, Zhang Z, Lu R and Lu J: Apigenin inhibits cell proliferation, migration, and invasion by targeting AKT in the A549 human lung cancer cell line. Anticancer Drugs 28(4): 446-456, 2017. PMID: 28125432. DOI: 10.1097/ CAD.0000000000000479

58 Yan X, Qi M, Li P, Zhan Y and Shao H: Apigenin in cancer therapy: Anticancer effects and mechanisms of action. Cell Biosci 7: 50, 2017. PMID: 29034071. DOI: 10.1186/s13578017-0179-x

59 Ozbey U, Attar R, Romero MA, Alhewairini SS, Afshar B, Sabitaliyevich UY, Hanna-Wakim L, Ozcelik B and Farooqi AA: Apigenin as an effective anticancer natural product: Spotlight on trail, Wnt/beta-catenin, JAK-STAT pathways, and microRNAs. J Cell Biochem, 2018. PMID: 30278099. DOI: 10.1002/ jcb. 27575

60 Gao AM, Zhang XY, Hu JN and Ke ZP: Apigenin sensitizes hepatocellular carcinoma cells to doxorubic through regulating mir-520b/ATG7 axis. Chem Biol Interact 280: 45-50, 2018. PMID: 29191453. DOI: 10.1016/j.cbi.2017.11.020

Received August 14, 2019

Revised September 30, 2019 Accepted October 8, 2019 